\title{
Effects of Alkyl Amide Solvents on the Dispersion of Single Wall Carbon Nanotubes
}

\author{
Brian J. Landi, ${ }^{\dagger}$ Herbert J. Ruf, ${ }^{\dagger}$ James J. Worman, ${ }^{\ddagger}$ and Ryne P. Raffaelle ${ }^{\dagger *}$ \\ NanoPower Research Laboratories (NPRL), Rochester Institute of Technology, Rochester, NY, \\ 14623 and Department of Chemistry, Rochester Institute of Technology, Rochester, NY, 14623
}

Email: rprsps@rit.edu

${ }^{\dagger}$ NPRL

${ }^{\ddagger}$ Department of Chemistry

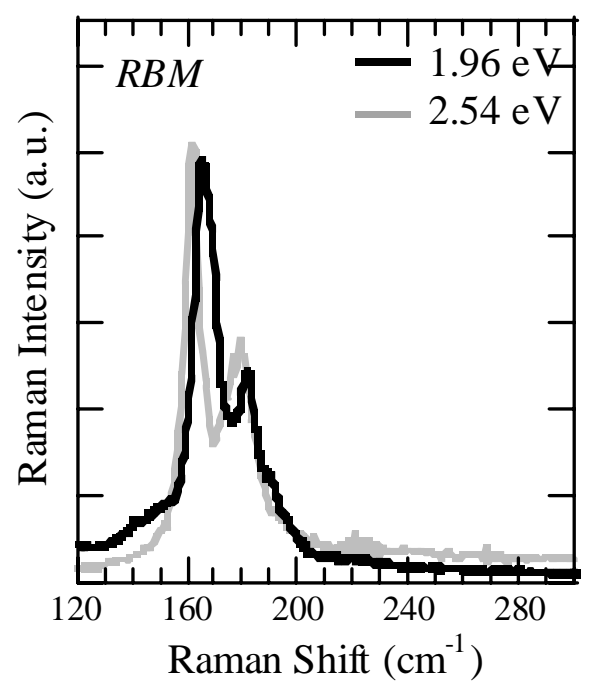

Figure S1. Shown is the Raman spectrum of the radial breathing mode (RBM) for lasergenerated SWNTs, which is used to calculate the diameter distribution. The use of two incident laser energies allows for the determination of SWNT type, i.e. semiconducting vs. metallic. For this diameter range, the $1.96 \mathrm{eV}$ laser probes predominantly metallic SWNTs and the $2.54 \mathrm{eV}$ laser resonantly enhances the semiconducting types. The data corresponds to SWNT diameters for both metallic and semiconducting types of $\sim 1.2-1.4 \mathrm{~nm}$ based on the following relation between Raman shift $\left(\omega_{\mathrm{RBM}}, \mathrm{cm}^{-1}\right)$ and diameter $(\mathrm{d}, \mathrm{nm}):^{25}$

$$
\omega_{R B M}=(224 / d)+14
$$

